This is the final peer-reviewed accepted manuscript of:

C. Orozco, S. Lilla, A. Borghetti, F. Napolitano and F. Tossani, "An ADMM Approach for Day-Ahead Scheduling of a Local Energy Community," 2019 IEEE Milan PowerTech, Milan, Italy, 2019, pp. 1-6.

The final published version is available online at:

https://doi.org/10.1109/PTC.2019.8810578

Rights / License:

The terms and conditions for the reuse of this version of the manuscript are specified in the publishing policy. For all terms of use and more information see the publisher's website. 


\title{
An ADMM Approach for Day-Ahead Scheduling of a Local Energy Community
}

\author{
C. Orozco, S. Lilla, A.Borghetti, F. Napolitano, F. Tossani \\ Department of Electrical, Electronic and Information Engineering \\ University of Bologna, Italy
}

camilo.orozco2@unibo.it, stefano.lilla3@unibo.it, alberto.borghetti@unibo.it, fabio.napolitano@unibo.it, fabio.tossani@unibo.it

\begin{abstract}
The paper deals with the day-ahead operational planning of a grid-connected local energy community (LEC) consisting of several prosumers each equipped with generating units, loads and battery energy storage units. The prosumers are connected to the same low-voltage distribution network. In order to preserve, as much as possible, the confidentiality of the features and forecast of prosumers' equipment, the problem is addressed by designing a specific distributed procedure based on the alternating direction method of multipliers (ADMM). The distributed procedure provides the scheduling of the batteries to limit the balancing action of the external grid. Results obtained for various case studies are compared with those obtained by a centralized approach. The values of the objective function, the profiles of the power exchanged with the utility grid and the profile of the energy stored in the batteries provided by the distributed approach are in close agreement with those calculated by the centralized one.
\end{abstract}

Index Terms--alternating direction method of multipliers, distributed optimization, energy management, local energy community, mixed integer programming, mixed integer quadratic programming.

\section{INTRODUCTION}

A local energy community (LEC) is a set of residential or small industrial sites each acting as a prosumer, being equipped, in general, with generation units, battery energy storage (BES) units and loads. All the prosumers are connected to the same low-voltage (LV) distribution network, which is the internal network of the LEC and it is connected to the medium-voltage (MV) external utility grid.

In a LEC, each prosumer uses the available energy resources in cooperation with the others to minimize the energy procurement costs. Due to the difference between the price of the energy supplied by the utility grid and the price paid to the local energy production, the power exchanges with the utility grid are reduced.

The operation of a LEC needs the implementation of an energy management system (EMS) for the optimal scheduling of the available resources [1]. This paper focuses on an algorithm for the day-ahead scheduling of the BES units. We assume that all the generation units of the LEC are photovoltaic
(PV) panels and the effects of power loss in the internal network can be neglected.

The developed scheduling algorithm is based on the alternating direction method of multipliers (ADMM). With respect to a centralized approach, the adoption of distributed approaches, as the ADMM, is preferable for the solution of the problem considered, since it reduces the need for each prosumer to communicate all the features and forecasts of the its own units and loads to the other prosumers or to a coordinating unit (e.g., [2]-[5]).

This paper does not address the issues of the uncertainty associated with the day-ahead forecasts of PV production and load profiles, as accomplished, for instance, in [6].

ADMM is one of the most frequently adopted consensus methods [7] and it has been recently investigated also for the solution of scheduling problems in microgrids (e.g., [8], [9], and references therein). In particular, both [8] and [9] deals with similar multi-microgrid systems as the one considered in this paper, with the presence of local generation, BES units and the possibility to exchange energy with an external utility grid. Moreover, [9] addresses the uncertainty of renewable energy, load consumption, and energy prices through a robust optimization approach.

The specific characteristics of the method proposed in this paper are:

- the ADMM approach is compared with a mixed integer linear programing (MILP) model of the centralized approach that includes the same constraints;

- both the centralized approach and distributed one allow the effective scheduling of the storage systems owned by the various prosumers;

- the structure of the proposed scheduling functions is consistent with the billing procedure and the metering units installed in the LEC.

The structure of the paper is the following. Section II is devoted to the description of a centralized approach based on a MILP model. Section III presents the proposed distributed

This work is supported in part by the Italian Ministry of Economic Development in the framework of CERSE research project PODCAST and by the European Union's Horizon 2020 research and innovation programme under the Marie Sklodowska-Curie grant agreement No 675318 (INCITE) and the Electronic Components and Systems for European Leadership Joint Undertaking under grant agreement No 737434 (CONNECT). 
approach based on the ADMM method. Section IV illustrates the results of the numerical tests. Section V concludes the paper.

\section{PROBLEM FormulATION - CENTRALIZED APPROACH}

Fig. 1 illustrates the LEC scheme. The point of common coupling (PCC) with the utility grid is represented by the LV side of the distribution transformer. The grid meter $\mathrm{M}_{\mathrm{g}}$, positioned at the PCC, is bidirectional to measure the net energy exchanged by the LEC with the utility grid in each time interval (bought or sold).

Moreover, for the implementation of the distributed optimization approach, each prosumer $i$ is equipped with a local bidirectional meter $M_{i}$ that measures the energy that the specific prosumer exchanges (sells or buys) with the internal network in each time interval. We assume that the meters provide the value of the energy exchanged every 15-min interval, considering the flow direction. The sign of the energy value identifies the average behavior of the prosumer during the 15-min interval: for example, a positive value means that the prosumer acts as consumer and a negative value means that the prosumer acts as a producer.

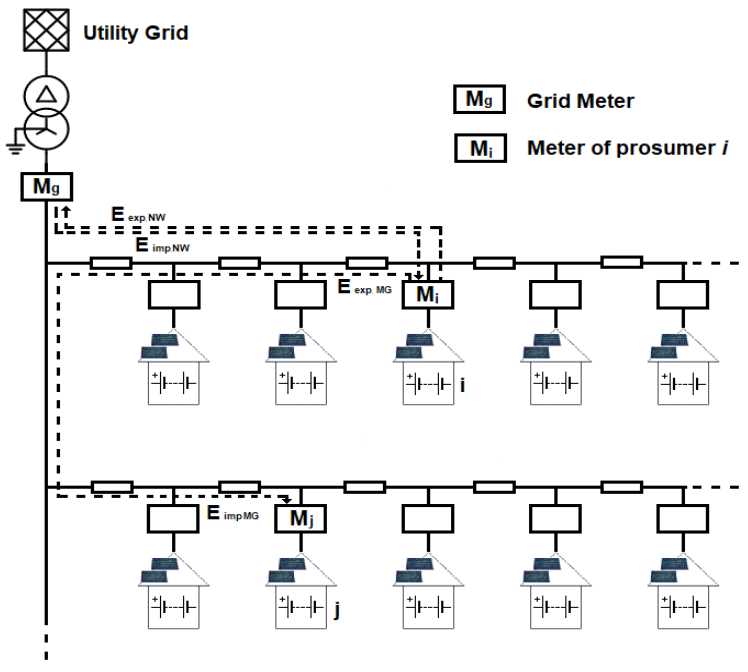

Figure 1. Scheme of the LEC.

The day ahead scheduling dealt with in this paper provides a plan of the optimal use of the LEC energy resources during the next day, with particular reference to the BES units, and calculates the prices of the energy transactions between prosumers. The prices of the exchanges with the utility grid are assumed to be fixed.

The electricity billing procedure can be described as follows:

a) in each time interval, if the LEC buys energy from the utility grid (measured by $\mathrm{M}_{\mathrm{g}}$ ), the relevant cost is allocated to each consumer $i$ (i.e., a prosumer that absorbs energy in that time interval) proportionally to the ratio of its consumption measured by $\mathrm{M}_{\mathrm{i}}$ and the total consumption in the LEC, i.e., the sum of the measured energies of all the prosumers acting as consumers;

b) if the LEC sells energy to the utility grid (measured by
$\mathrm{M}_{\mathrm{g}}$ ), the relevant revenue is allocated to each producer $j$ (i.e., a prosumer that produces energy in excess of the local load in that time interval) proportionally to the contribution of $j$ to the total LEC production, i.e., the ratio between the energy measured by $\mathrm{M}_{\mathrm{j}}$ and the sum of the measurements of all the prosumers acting as producers;

c) each consumer $i$ is also charged of the energy bought from the local producers, i.e. the energy given by the difference between the measurement of $\mathrm{M}_{\mathrm{i}}$ and the energy allocated to consumer $i$ in step a). The corresponding revenue of producer $j$ is estimated proportionally to the contribution of $j$ to the total LEC production as in step b). The day ahead procedure calculates the prices of each prosumer $j$ that produces energy.

By denoting as $\Omega=\{1,2, \ldots, N\}$ the set of prosumers and as $T=\left\{1,2, \ldots, t_{\text {end }}\right\}$ the set of $9615-$ min periods of the optimization horizon, the Objective Function (OF) (1) minimizes the total cost associated with the power exchanged with the utility grid in the entire time horizon: parameters $\pi_{b u y}^{t}$ and $\pi_{\text {sell }}^{t}$ are the prices (in $€ / \mathrm{kWh}$ ) of the energy bought from and sold to the utility grid, respectively; $P_{\text {buy_Grid } i}^{t}$ and $P_{\text {sell_Grid } i}^{t}$ are the average power bought from and sold to the utility grid (in $\mathrm{kW}$ ) in time interval $t$, respectively; parameter $\Delta t$ is the time step $(0.25 \mathrm{~h})$.

$$
O F=\min \sum_{\substack{t \in T \\ i \in \Omega}}\left(\pi_{\text {buy }}^{t} P_{\text {buy_Grid } i}^{t}-\pi_{\text {sell }}^{t} P_{\text {sell_Grid } i}^{t}\right) \Delta t
$$

The constraints are:

$$
\begin{aligned}
& \sum_{\substack{j \in \Omega \\
j \neq i}} P_{\text {buy } j, i}^{t} \Delta t-\sum_{\substack{j \in \Omega \\
j \neq i}} P_{\text {sell } i, j}^{t} \Delta t=0 \quad t \in T \quad i \in \Omega \\
& P_{\mathrm{G} i}^{t}+P_{\mathrm{BES} \_ \text {dis } i}^{t}+P_{\text {buy_Grid } i}^{t}+\sum_{\substack{j \in \Omega \\
j \neq i}} P_{\text {buy } i, j}^{t}= \\
& t \in T \quad i \in \Omega \\
& P_{\mathrm{D} i}^{t}+P_{\mathrm{BES} \_ \text {ch }, i}^{t}+P_{\text {sell_Grid } i}^{t}+\sum_{\substack{j \in \Omega \\
j \neq i}} P_{\text {sell } i, j}^{t} \\
& \begin{cases}P_{\text {buy_Grid } i}^{t}=0 \text { and } P_{\text {buy } i, j}^{t}=0 \text { if } u_{i}^{t}=0 & u_{i}^{t} \in\{1,0\} \\
P_{\text {sell_Grid } i}^{t}=0 \text { and } P_{\text {sell } i, j}^{t}=0 \text { if } u_{i}^{t}=1 & i, j \in \Omega\end{cases} \\
& 0 \leq P_{\text {buy_Grid } i}^{t} \leq P_{\text {buy } i}^{\max } \quad 0 \leq P_{\text {sell_Grid } i}^{t} \leq P_{\text {sell } i}^{\max } \quad t \in T \quad i \in \Omega \\
& 0 \leq P_{\text {buy } i, j}^{t} \leq P_{\text {buy } i}^{\max } \quad 0 \leq P_{\text {sell } i, j}^{t} \leq P_{\text {sell } i}^{\max } t \in T \quad i, j \in \Omega \\
& E_{\mathrm{BES} i}^{t}=E_{\mathrm{BES} i}^{t-1}+\left(P_{\mathrm{ch} i}^{t} \eta_{\mathrm{ch}}-P_{\mathrm{dis} i}^{t} / \eta_{\mathrm{dis}}\right) \Delta t \quad \begin{array}{c}
i \in \Omega \\
t \in T, t>1
\end{array} \\
& \left\{\begin{array}{l}
E_{\mathrm{BES} i}^{t=1}=E_{\mathrm{max}, i}+\left(P_{\mathrm{BES} i}^{t=1} \eta_{\mathrm{ch}}-P_{\mathrm{BES} i}^{t=1} / \eta_{\mathrm{dis}}\right) \Delta t \quad i \in \Omega \\
E_{\mathrm{BES} i}^{t_{\mathrm{end}}}=E_{\mathrm{BES} i}^{\max } \quad i \in \Omega
\end{array}\right. \\
& \left\{\begin{array}{lll}
P_{\mathrm{ch} i}^{t}=0 & \text { if } \quad u_{\mathrm{BES} i}^{t}=0 & u_{\mathrm{BES} i}^{t} \in\{1,0\} \\
P_{\mathrm{dis} i}^{t}=0 \text { if } \quad u_{\mathrm{BES} i}^{t}=1 & i \in \Omega
\end{array}\right. \\
& 0 \leq P_{\mathrm{BES} \_ \text {dis } i}^{t} \leq P_{\mathrm{BES} i}^{\max } \quad 0 \leq P_{\mathrm{BES} \_ \text {ch } i}^{t} \leq P_{\mathrm{BES} i}^{\max } \quad t \in T \quad i \in \Omega \\
& E_{\mathrm{BES} i}^{\min } \leq E_{\mathrm{BES} i}^{t} \leq E_{\mathrm{BES} i}^{\max } t \in T \quad i \in \Omega
\end{aligned}
$$

Constraint (2) represents the equilibrium between the 
energy bought from producer $i$ by the other prosumers and the energy sold by producer $i$ to the other prosumers in time interval $t$. The Lagrangian multiplier $\lambda_{i}^{t}$ associated to (2) is the price of the energy sold by $i$ in time interval $t$. With this formulation of constraint (2), the price is independent of the buying prosumer, according to the cooperative behavior of the LEC participants.

Constraint (3) represents the power balance for the $i$-th prosumer: parameters $P_{\mathrm{G} i}^{t}$ and $P_{\mathrm{D} i}^{t}($ in $\mathrm{kW}$ ) are the average $\mathrm{PV}$ power generation and demand of $i$ in time interval $t$, respectively; non-negative variables $P_{\mathrm{ch} i}^{t}$ and $P_{\mathrm{dis} i}^{t}$ (in $\mathrm{kW}$ ) are the charging and discharging average power in the BES unit of prosumer $i ; P_{\text {buy } i, j}^{t} \Delta t$ and $P_{\text {sell } i, j}^{t} \Delta t$ (in $\mathrm{kWh}$ ) are the energy bought by $i$ from $j$ and sold by $i$ to $j$, in time interval $t$, respectively.

Indicator constraints (4), with binary variable $u_{\mathrm{i}}^{t}$, are used to avoid simultaneous purchase and selling by the same prosumer.

The possibility of prosumer $i$ to buy or sell energy is limited by constraints (5) and (6) where $P_{\text {sell } i}^{\max }$ is the largest value between 0 and $P_{\mathrm{G} i}^{t}-P_{\mathrm{D} i}^{t}+P_{\mathrm{BES} i}^{\max }$, and $P_{\mathrm{buy} i}^{\max }$ is the largest value between 0 and $P_{\mathrm{D} i}^{t}-P_{\mathrm{G} i}^{t}+P_{\mathrm{BES} i}^{\max } . P_{\mathrm{BES} i}^{\max }$ is the maximum power output of the BES unit of prosumer $i$.

For each storage, the state of the energy $(S o E)$ is defined by (7) and (8), where $E_{\mathrm{BES} i}^{t}$ is the $S o E$ at time $t$ (in $\mathrm{kWh}$ ) and $\eta_{\mathrm{ch}}$, $\eta_{\text {dis }}$ are the battery efficiencies during charge and discharge. In (8) we assume that BES units are fully charged at the beginning and at the end of the day, where $E_{\mathrm{BES} i}^{\max }$ is the size of the $i$-th storage.

The power during charge and discharge is limited by parameter $P_{\mathrm{BES}}^{\max }$ in constraint (10). The $\operatorname{SoE}\left(E_{\mathrm{BES} i}^{t}\right)$ is bounded between the minimum level $E_{\mathrm{BES} i}^{\min }$ and $E_{\mathrm{BES} i}^{\max }$ by constraint (11). In order to prevent simultaneous charge and discharge of the batteries, indicator constraints (9) with binary variable $u_{\mathrm{BES}}^{t}$ are included.

In the literature, more accurate MILP models of the BES are described (e.g., in [6], [10] and [11]) that can replace the simple model represented by (7)-(11).

As mentioned, in this preliminary model the losses and the limitations in the internal network of the LEC are disregarded. Therefore, the calculation of bus voltages and reactive power flows is not included in the model.

\section{PROBLEM FORMULATION - DistRIBUTED APPROACH}

The optimization is iteratively carried out by each prosumer $k$. At each ADMM iteration, the energy bought or sold by each prosumer in every time $t$ is made known to all the prosumers. These values are considered as parameters in the optimization problem solved by prosumer $k$ at the current iteration and they are denoted by a hat in the model described in this section.
The objective function of prosumer $k$ is

$$
O F_{k}=\min \sum_{t \in T}\left[\begin{array}{l}
\pi_{\text {buy }}^{t} P_{\text {buy_Grid } k}^{t} \Delta t-\pi_{\text {sell }}^{t} P_{\text {sell_Grid } k}^{t} \Delta t+ \\
\sum_{\substack{j \in \Omega \\
j \neq k}} \lambda_{j}^{t} P_{\text {buy } k, j}^{t} \Delta t-\lambda_{k}^{t} \sum_{\substack{j \in \Omega \\
j \neq k}} P_{\text {sell } k, j}^{t} \Delta t+\ell_{k}^{t}
\end{array}\right]
$$

where

$$
\ell_{k}^{t}=m \cdot \rho \cdot\left[\sum_{\substack{j \in \Omega \\ j \neq k}}\left(\hat{P}_{\text {buy } j, k}^{t}-P_{\text {sell } k, j}^{t}\right)^{2}+\sum_{\substack{j \in \Omega \\ j \neq k}}\left(P_{\text {buy } k, j}^{t}-\hat{P}_{\text {sell } j, k}^{t}\right)^{2}\right]
$$

Equation (12) is obtained by the decomposition for each prosumer $k$ of the Lagrangian that incorporates $O F$ (1) and constraints (2), each multiplied by the relevant Lagrange multiplier $\lambda_{i}^{t}$, augmented by $\ell_{k}^{t}$, namely, the squared norm of the same constraints multiplied by positive penalty parameter $\rho$ and fixed scale factor $m$, as shown in (13).

$O F_{k}$ can be seen as the summation of the costs of the energy bought by prosumer $k$ from the utility grid at price $\pi_{\text {buy }}^{t}$ and from the other prosumers at prices $\lambda_{j}^{t}$ minus the sum of the revenues due to the energy sold by prosumer $k$ to the utility grid at price $\pi_{\text {sell }}^{t}$ and to the other prosumers at price $\lambda_{k}^{t}$.

Once the procedure converges, the additional term $\ell_{k}^{t}$ is zero and the value $O F$ for the whole system is the sum of the ones solved for each prosumer $k$ :

$$
O F=\sum_{k \in \Omega} O F_{k}
$$

The optimization problem of prosumer $k$ includes constraints (3)-(11) for $i=k$.

Moreover, the convergence of the ADMM procedure is improved if the following constraints are added starting from the second iteration:

$$
\begin{aligned}
& P_{\text {sell } k, j}^{t} \leq \hat{P}_{\text {buy_Grid } j}^{t}+\sum_{\substack{i \in \Omega \\
i \neq j}} \hat{P}_{\text {buy } j, i}^{t} \quad t \in T \quad k, j \in \Omega \\
& P_{\text {buy } k, j}^{t} \leq \hat{P}_{\text {sell_Grid } j}^{t}+\sum_{\substack{i \in \Omega \\
i \neq j}} \hat{P}_{\text {sell } j, i}^{t} \quad t \in T \quad k, j \in \Omega
\end{aligned}
$$

At each iteration $v$, after the solution of all the optimization problems, one for each prosumer $k$, the ADMM includes the update of Lagrangian multipliers $\lambda_{k}^{t}$ and penalty parameter $\rho$. This update may be performed by using a distributed ledger avoiding the presence of a central coordinating unit.

Let $r_{k}^{v}$ be the primal residual term for prosumer $k$, equal to the vector of dimension $T$ with elements

$$
r_{k}^{t}=\sum_{\substack{j \in \Omega \\ j \neq k}} P_{\text {buy } j, k}^{t}-\sum_{\substack{j \in \Omega \\ j \neq k}} P_{\text {sell } k, j}^{t}
$$

the $T$ dimensional vector of Lagrangian multipliers $\lambda_{k}^{v}$, with elements $\lambda_{k}^{t}$, is updated as:

$$
\lambda_{k}^{v+1}=\lambda_{k}^{v}+2 m \cdot \rho \cdot r_{k}^{v}
$$


and the procedure is repeated until the absolute value of each residual $r_{k}^{t}$ becomes equal or lower than tolerance $\varepsilon$ (which is assumed to be $5 \mathrm{~W}$ in all the numerical tests of this paper), i.e., until $\left|r_{k}^{t}\right| \leq \varepsilon$. At the beginning of the ADMM procedure, prices $\lambda_{k}^{t}$ are initialized to be equal to $1 / 2\left(\pi_{\text {buy }}^{t}+\pi_{\text {sell }}^{t}\right)$.

The penalty parameters are updated as follows [7]:

$$
\rho^{v+1}= \begin{cases}2 \rho^{v} & \left\|r_{k}^{v}\right\|_{2}>10\left\|s_{k}^{v}\right\|_{2} \\ \rho^{v} / 2 & \left\|s_{k}^{v}\right\|_{2}>10\left\|r_{k}^{v}\right\|_{2} \\ \rho^{v} & \text { otherwise }\end{cases}
$$

where \|\|$_{2}$ is the Euclidian norm and the $T$ dimensional vector $s_{k}^{v}$ is the dual residual term

$$
s_{k}^{t}=\rho^{v}\left[\begin{array}{l}
\left(\sum_{j \in \Omega} P_{\text {sell } k, j}^{t}\right)^{v}-\left(\sum_{j \in \Omega} P_{\text {sell } k, j}^{t}\right)^{v-1} \\
+\left(\sum_{j \in \Omega} P_{\text {buy } k, j}^{t}\right)^{v}-\left(\sum_{j \in \Omega} P_{\text {buy } k, j}^{t}\right)^{v-1}
\end{array}\right]
$$

To accelerate the convergence, the initial value of $m$ equal to $5 \cdot 10^{-5}$ is multiplied by 10 when the maximum value of the total mismatch $r^{t}=\sum_{k}\left|r_{k}^{t}\right|$ becomes lower than $1 \mathrm{~kW}$, and further multiplied by 10 when $\max \left(\left|r_{k}^{t}\right|\right)<100 \mathrm{~W}$.

\section{NUMERICAL TESTS}

The models have been implemented in the AIMMS Developer modelling environment [12] and tested by using the Cplex V12.8 [13] solver on a 2-GHz Intel-i7 computer with 8 GB of RAM, running 64-bit Windows 10. The MILP solver is used for the centralized model and the MIQP (mixed integer quadratic programming) solver for the ADMM model.

The test system is composed of two LV feeders, each with five prosumers connected. Each prosumer has a PV unit and a local load. As mentioned, all the calculations refer to a time window of 1 day, split in 96 periods of 15 min each.

Now, we describe the inputs data. The adopted load profiles are shown in Fig. 2. For the PV generation, we have assumed the profile of the ratio between power output and panel surface shown in Fig. 3. The area of the PV panels is given in Table I.

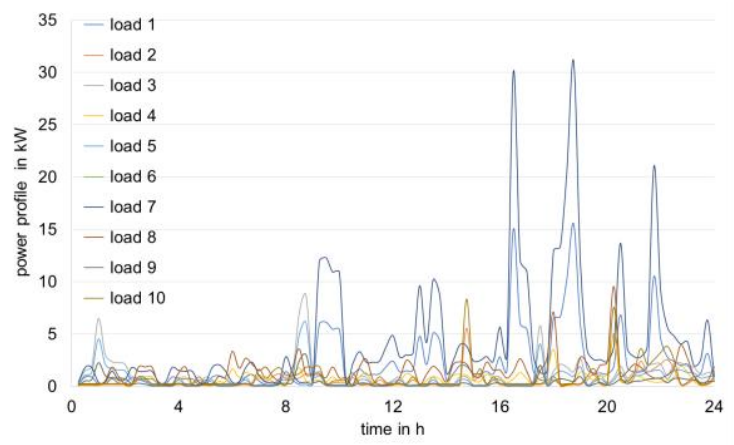

Figure 2. Load profile for each prosumer.

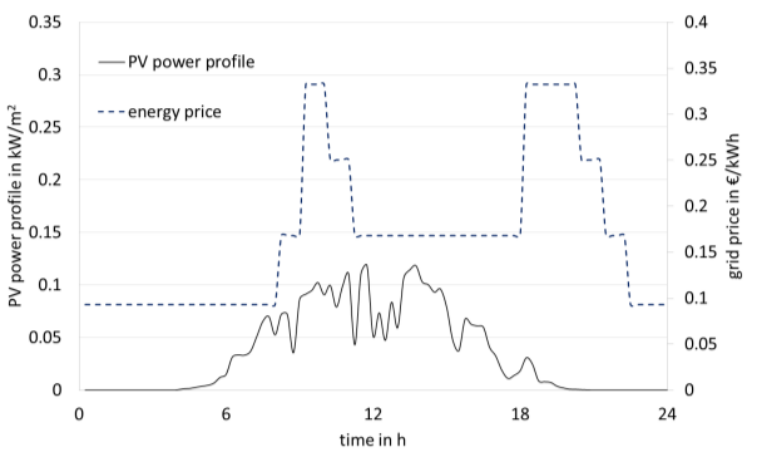

Figure 3. Profile of the PV production and grid purchase price.

TABLE I. PV PANEL SURFACE FOR EACH PROSUMER

\begin{tabular}{c|c|c|c|c|c|c|c|c|c|c}
\hline \hline prosumer & 1 & 2 & 3 & 4 & 5 & 6 & 7 & 8 & 9 & 10 \\
\hline area $\left(\mathrm{m}^{2}\right)$ & 32 & 14 & 21 & 32 & 28 & 14 & 42 & 32 & 14 & 42 \\
\hline \hline
\end{tabular}

Fig. 3 also shows the price profile of the energy bought from the utility grid $\pi_{\text {buy }}^{t}$. We assume that the price of the energy sold by the LEC to the utility grid, i.e. $\pi_{\text {sell }}^{t}$, is half of $\pi_{\text {buy }}^{t}$.

We repeat the calculations two times, once assuming the system without BES units and the other by assuming that each prosumer is also equipped with a BES unit. The adopted values of $E_{\mathrm{BES}}^{\max }$ are reported in Table II. The same values are also adopted for the corresponding maximum power output $P_{\mathrm{BES}}^{\max }$.

TABLE II. SIZES OF THE BES UNITS

\begin{tabular}{c|c|c|c|c|c|c|c|c|c|c}
\hline \hline prosumer & 1 & 2 & 3 & 4 & 5 & 6 & 7 & 8 & 9 & 10 \\
\hline size $(\mathrm{kWh})$ & 5 & 3 & 4 & 2 & 3 & 1 & 2 & 2 & 2 & 6 \\
\hline \hline
\end{tabular}

Now, we compare the solutions obtained by applying the centralized and the distributed models for both the case study with the BES units and the one without BES units. Table III compares the $O F$ values that are almost the same for the two approaches. The solution of the centralized model needs around $0.4 \mathrm{~s}$ without BES units and $7 \mathrm{~s}$ with BES units. The solution of the distributed model needs $40 \mathrm{~s} / 12$ iterations without BES units, and around $170 \mathrm{~s} / 26$ iterations with BES units. In the implemented ADMM procedure, the optimization problems of the prosumers are solved in sequence. Furthermore, we assume that the communication channels do not have delays or limitations. As expected, the computational effort decreases if a longer $\Delta t$ is adopted. For example, if $\Delta t=30 \mathrm{~min}$, the centralized approach needs around $0.2 \mathrm{~s}$ without BES units and $3.3 \mathrm{~s}$ with BES units, whilst the distributed model requires $25 \mathrm{~s}$ and $60 \mathrm{~s}$, respectively. The ADMM adoption is not justified by the computational time, but by its capability to reduce the amount of shared information.

TABLE III. COMPARISON BETWEEN CENTRALIZED AND ADMM

\begin{tabular}{c|c|c}
\hline \hline & \multicolumn{2}{|c}{ OF $(€)$} \\
& without BES & with BES \\
\hline Centralized & 26.58 & 17.84 \\
\hline ADMM & 26.58 & 17.98 \\
\hline \hline
\end{tabular}

To illustrate the convergence behavior of the ADMM procedure, Fig. 4 shows the average value of the primal 
residuals $r_{k}^{t}$ denoted by $R$ (in $\mathrm{W}$ ), the values of the augmented $O F$ (14) (in $€$ ) and the value of the part of $O F$ relevant to the energy bought from and sold to the utility grid, i.e. (1), at each iteration.

a)


b)

Figure 4. ADMM convergence (augmented $O F$, part of $O F$ corresponding to the power exchanged with the utility grid, average of primal residuals at each iteration): a) without BES units, b) with BES units.

a)
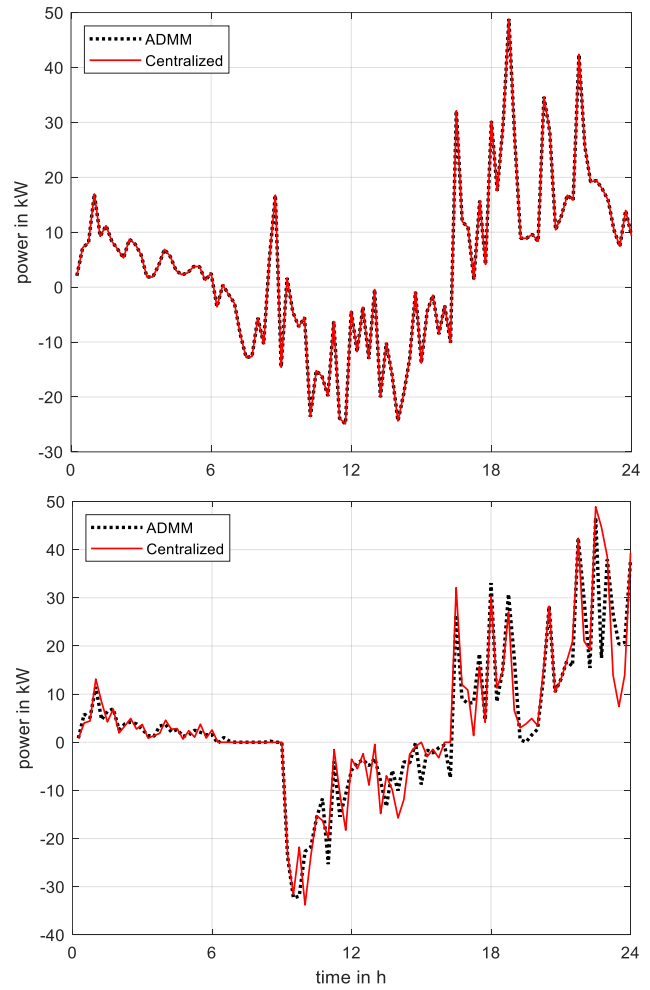

b)

Figure 5. Comparison of the profiles of the power exchanged with the utility grid obtained by using the centralized and the distributed approach: a) without BES units and $b$ ) with BES units. Positive values indicate imported power from the utility grid, negative values indicate exported power to the utility grid.
The comparison between the profiles of the power exchanged with the utility grid is shown in Fig. 5. As expected, the profiles are quite similar.

Fig. 6 shows the comparison between the profiles of the total energy contained in the BES units of the LEC.

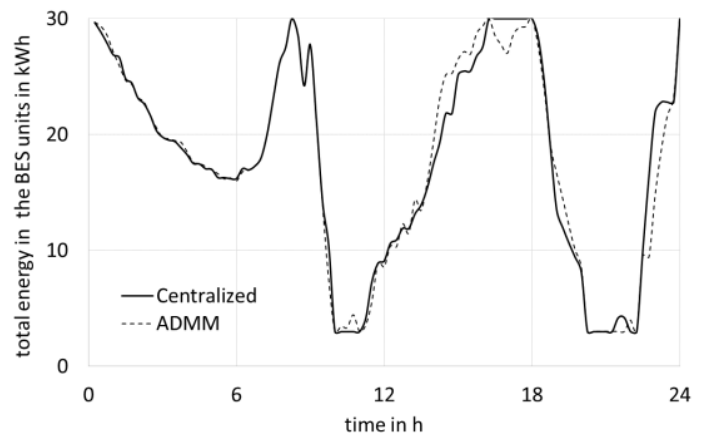

Figure 6. Comparison of the total energy in the batteries of the LEC obtained by the centralized and the distributed approach.

Fig. 7 and Fig. 8 show the energy prices $\lambda_{i}^{t}$ for the case of the system without and with BES units, respectively. As mentioned, for the case of the centralized model, the inferred prices correspond to the Lagrangian multiplier associated to constraint (2). The dotted lines correspond to the prices of the energy bought from and sold to the utility grid (i.e., $\pi_{\text {buy }}^{t}$ and $\pi_{\text {sell }}^{t}$ ), while the solid lines represent the transaction prices of the various prosumers when they sell energy to any other prosumer of the LEC.

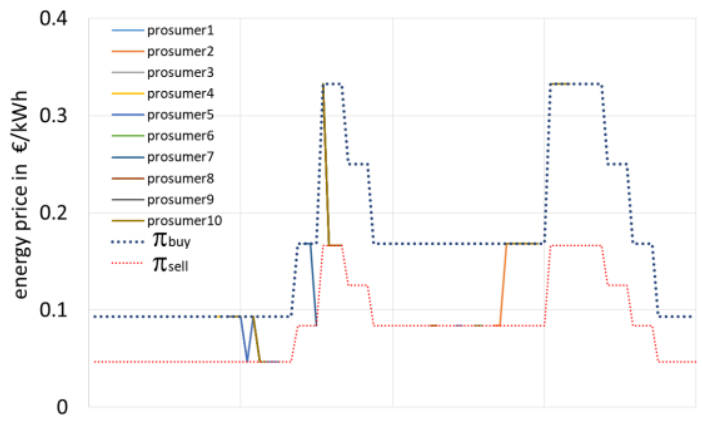

a)

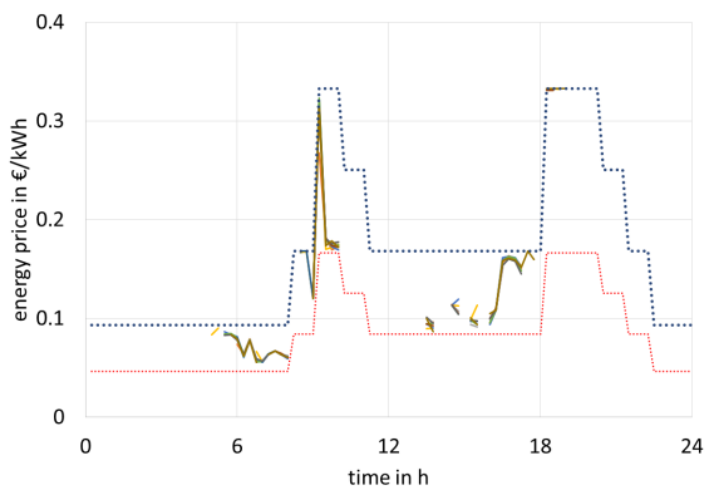

Figure 7. Energy prices of selling prosumers for the system without BES units: a) centralized model, b) last iteration of the ADMM procedure.

For the system without BES units, Fig. 7a shows that, according to the centralized model, the prices of the selling 
prosumers are equal to $\pi_{\text {buy }}^{t}$ when the LEC, as a whole, imports energy from the utility grid. Furthermore, the prices are equal to $\pi_{\text {sell }}^{t}$ when the LEC sells energy to the utility grid. Similar results are obtained at the last iteration of the ADMM procedure, in which the prices are updated by using (18).

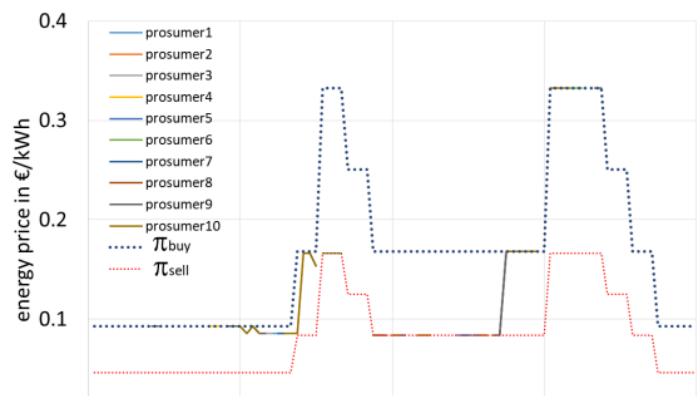

a)

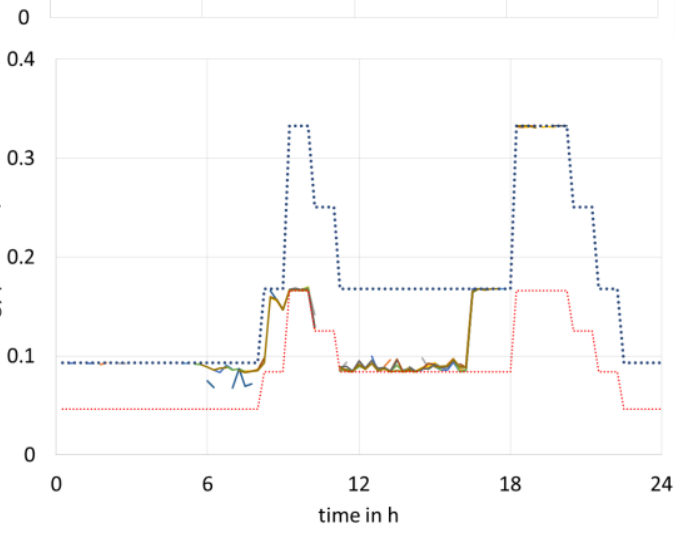

b)

Figure 8. Energy prices of selling prosumers for the system with BES units: a) centralized model, b) last iteration of the ADMM procedure.

For the case of the system with BES units, Fig. 8 compares the price profiles of each prosumers calculated by the centralized model (Fig. 8a) and at the last iteration of the ADMM procedure (Fig. 8b). The comparison of these results with Fig. 5 shows that the prices are significantly different from $\pi_{\text {buy }}^{t}$ or $\pi_{\text {sell }}^{t}$ only in the time intervals when there is limited exchange with the utility grid.

\section{CONCLUSION}

The paper has presented an optimization procedure for the day-ahead scheduling of a local energy community with generation, loads and battery storage systems.

The results obtained by using the proposed distributed optimization procedure based on the application of the alternating direction method of multipliers have been compared with those from a centralized approach based on a mixed integer linear programming model. The distributed approach has the advantage to reduce the information that each prosumer must share with the other prosumers.

Both centralized approach and the distributed one provide comparable results with an acceptable computational effort.

The values of the objective function, the profiles of the power exchanged with the utility grid and the profile of the energy stored in the batteries match.
The prices of each prosumer $i$ that sells to other prosumers of the community are calculated in different way in the two procedures. In the centralized procedure, the prices are the Lagrangian multipliers of the constraints stating the equality between the energy sold by a prosumer $i$ and the energy bought by the others prosumers from prosumer $i$. In the distributed procedure, the prices are updated at each iteration to reduce the mismatch between the energy sold by each prosumer $i$ and the energy bought by the other prosumers from prosumer $i$. Notwithstanding these differences, the profiles of the prices are similar for both the cases with and without BES units.

The structure of the day-ahead scheduling procedures is consistent with the billing scheme and the metering units of the LEC.

\section{ACKNOWLEDGMENT}

The authors thank Beatriz Ildefonso for her help in the model implementation. Special thanks to Prof. Carlo Alberto Nucci for helpful discussions during the development of this work.

\section{REFERENCES}

[1] B. Prasad, E. Koliou, J. Friege, R. A. Hakvoort, and P. M. Herder, "Energetic communities for community energy: a review of key issues and trends shaping integrated community energy systems," Renew. Sustain. Energy Rev., vol. 56, pp. 722-744, 2016.

[2] M. L. Di Silvestre, P. Gallo, M. G. Ippolito, and E. Riva Sanseverino, "A technical approach to the energy blockchain in microgrids," IEEE Trans. Ind. Informatics, vol. 14, no. 11, pp. 4792-4803, 2018.

[3] C. Long, J. Wu, C. Zhang, L. Thomas, M. Cheng, and N. Jenkins, "Peerto-peer energy trading in a community microgrid," in 2017 IEEE Power \& Energy Society General Meeting, 2017, pp. 1-5.

[4] A. Paudel, K. Chaudhari, C. Long, and H. Gooi, "Peer-to-Peer Energy Trading in a Prosumer Based Community Microgrid: A GameTheoretic," IEEE Trans. Ind. Electron., vol. PP, no. c, p. 1, 2018.

[5] Y. Zhao, J. Yu, M. Ban, Y. Liu, and Z. Li, "Privacy-preserving economic dispatch for an active distribution network with multiple networked microgrids," IEEE Access, vol. 6, pp. 38802-38819, 2018.

[6] C. Orozco, A. Borghetti, S. Lilla, G. Pulazza, and F. Tossani, "Comparison between multistage stochastic optimization programming and Monte Carlo simulations for the operation of local energy systems," in IEEE International Conference on Environment and Electrical Engineering (EEEIC), 2018.

[7] S. Boyd, N. Parikh, E. Chu, B. Peleato, and J. Eckstein, Distributed optimization and statistical learning via the alternating direction method of multipliers. Foundations and Trends in Machine Learning, 2011.

[8] Y. Zheng, Y. Song, D. J. Hill, and Y. Zhang, "Multiagent System Based Microgrid Energy Management via Asynchronous Consensus ADMM," IEEE Trans. Energy Convers., vol. 33, no. 2, pp. 886-888, 2018.

[9] Y. Liu, Y. Li, H. B. Gooi, J. Ye, H. Xin, X. Jiang, and J. Pan, "Distributed robust energy management of a multimicrogrid system in the real-time energy market," IEEE Trans. Sustain. Energy, vol. 10, no. 1, pp. 396406, 2019.

[10] A. Sakti, K. G. Gallagher, N. Sepulveda, C. Uckun, C. Vergara, F. J. de Sisternes, D. W. Dees, and A. Botterud, "Enhanced representations of lithium-ion batteries in power systems models and their effect on the valuation of energy arbitrage applications," J. Power Sources, vol. 342, pp. 279-291, 2017.

[11] C. Bordin, H. O. Anuta, A. Crossland, I. L. Gutierrez, C. J. Dent, and D. Vigo, "A linear programming approach for battery degradation analysis and optimization in offgrid power systems with solar energy integration," Renew. Energy, vol. 101, pp. 417-430, 2017.

[12] M. Roelofs and J. Bisschop, AIMMS Language Reference. 2013.

[13] IBM/ILOG, Introducing IBM ILOG CPLEX Optimization Studio V12.8. 2018. 